\title{
COMPUTATIONAL CONCEPTUAL DISTANCES IN COMBINATIONAL CREATIVITY
}

\author{
J. Han ${ }^{1, \bigotimes}$, M. Hua ${ }^{2}$, D. Park ${ }^{2}$, P. Wang ${ }^{2}$ and P. R. N. Childs ${ }^{2}$ \\ ${ }^{1}$ University of Liverpool, United Kingdom, ${ }^{2}$ Imperial College London, United Kingdom \\ $\bigotimes$ Ji.Han@liverpool.ac.uk
}

\section{Abstract}

Combinational creativity can play a significant role in supporting designers to produce creative ideas during the early stages of new product development. This paper explores conceptual distances in combinational creativity from computational perspectives. A study conducted indicates that different computational measurements show different conceptual distance results. However, the study suggests far-related ideas could lead to outcomes that are more creative than closely-related ones. This paper provides useful insights into exploring future computational design support tools.

Keywords: design creativity, idea generation, computational design methods, combinational, conceptual distance

\section{Introduction}

Creativity is essential to new product design and development (Sarkar and Chakrabarti, 2011). It is also a natural element of the deign process (Demirkan and Afacan, 2012). Designers from various areas are required to be creative to generate new and useful solutions or ideas (Crilly and Cardoso, 2017). Creative ideas, which are novel, useful and surprising (Han et al., 2019a), often lead to creative and successful designs (Chiu and Shu, 2012). However, it is challenging to produce creative design ideas.

Combinational creativity is an approach for producing creative ideas through unfamiliar combinations of familiar ideas (Boden, 2004; Boden, 2009). In other words, it generates new ideas through associating ideas that are previously not or indirectly related. Combinational designs, which are originated by combining different ideas, are outcomes of employing combinational creativity in design. Combinational creativity has been claimed to be one of the easiest approaches for humans to produce creative ideas, as it is a natural feature of human associative memory. Also, it is considered the best approach to fully utilise abundant knowledge to generate creative ideas (Yang and Zhang, 2016). However, combining ideas might lead to challenges in terms of the time required to produce and evaluate all the possible combinations, which is called 'combinational explosion' (Simonton, 2017).

Recently, a number of studies have explored the use of combinational creativity to support creative idea generation in design. Han et al. $(2019 \mathrm{~b}, 2019 \mathrm{c})$ have proposed three driven approaches to combinational creativity: problem-, similarity- and inspiration-driven, and indicated that the problemdriven approach could lead to more creative and novel ideas; Taura et al. (2007) and Nagai et al. (2009) have explored different linguistic interpretation methods of combinational phrases for producing creative ideas. Computationally, several tools and approaches have been explored for supporting designers in creative idea generation. For example, Georgiev et al. (2017) proposed an 
approach to create new scenes through synthesising existing scenes to support new product design; Han et al. (2018a) developed a tool that produces combinational prompts in image- and text-forms; Chen et al. (2019) came up with an artificial intelligence method to generate synthesised images, by using a generative adversarial network, to stimulate designers' creative minds.

Conceptual distances between ideas play an essential role in forming combinational ideas. A recent study by Han et al. (2018b) has provided insights into how design experts understand conceptual distances in combinational creativity, of which far-related ideas are more beneficial than closelyrelated ones. However, a computational understanding of conceptual distances has not yet been explored in combinational creativity.

This paper is aimed at exploring conceptual distances from a computational perspective. The following section provides a review of related studies on conceptual distances and combinational creativity. In section 3, a study employing computational conceptual distance measurement tools is conducted to investigate computational conceptual distances in combinational creativity. The results of the study are compared with the ones from Han et al. (2018b), and followed by further analysis as well as discussion in section 4 and conclusions in section 5 .

\section{Related work}

\subsection{Conceptual distance}

Conceptual distance is a measurement of the distance between two concepts or two sets of concepts (Gray and Rumpe, 2019). In linguistics, semantic distance is described as a measurement of the degree of semantic association between the meanings of two language units, such as words, concepts, phrases and sentences (Cooper, 2000; Mohammad, 2008). Conceptual distance and semantic distance are often used interchangeably in literature. Therefore, conceptual distance and semantic distance both refer to measuring the distance between two concepts in this paper.

There are two types of semantic distance, which are semantic similarity and semantic relatedness (Budanitsky and Hirst, 2006; Resnik, 1999). Semantic similarity indicates the degree of resemblance between two concepts, which plays a significant role in knowledge processing, objects classifying, and concepts forming (Tversky, 1977). It measures how alike two concepts are (Lofi, 2015). For instance, "donkey" is semantically similar to "horse". Semantic relatedness refers to the degree to which two terms are associated via various semantic relationships (Salahli, 2009). It quantifies the relatedness of terms concerning other relations, such as functional and hyponymy (Lofi, 2015). For example, "harness" is semantically related, but not similar, to "horse". Although semantic distance, semantic similarity and semantic relatedness are not identical, they are often used interchangeably in literature. It is challenging for both humans and computers to differentiate the terms (Lofi, 2015; Salahli, 2009).

In design research, conceptual distance is often discussed within the context of analogy. Analogy is considered a specific form of combinational creativity projecting knowledge from the source domain to the target domain by exploring a shared conceptual space (Boden, 2009; Ward, 2011). Conceptual distance is conventionally considered a function of the degree of surface and structural similarity (Ozkan and Dogan, 2013). Gentner and Smith (2012) described surface similarity as "likeness based only on similar objects and background context between two domains/situations, without a common relational structure", and structural similarity as "likeness based on relations common to both domains or situations (whether or not the objects in the two systems resemble each other)". Blanchette and Dunbar (2000) defined surface similarity as "the resemblance between the objects in the source and target and their properties", and structural similarity as "the resemblance in the underlying systems of relations between the elements of the sources and the elements of the target". Surface similarity indicates a close relation, while structural similarity indicates a far relation. A tripartite classification has been proposed by Ward (1998) to identify different conceptual distances, which are "same conceptual domain", "related, though non-identical domains", and "wildly discrepant domains".

Conceptual distance plays a significant role in the design process, especially idea generation and creativity. Lopez et al. (2011) indicated that distant analogies have the potential in leading to more creative designs, due to more abstractions. However, Chan et al. (2015) suggested that design ideas produced from citing conceptually further sources are less creative than the ones produced from citing 
closer sources. In the study conducted by Ozkan and Dogan (2013), although expert designers are more likely to construct structural similarity, they prefer close domains in analogical reasoning due to design process-related considerations. Novices establish only surface similarity, but prefer far domains. Fu et al. (2013) claimed that "close" and "far" are contextual and relative terms. Thereby, it is challenging to come up with a detailed definition of "close" and "far" for conceptual distances. In this paper, we describe two concepts or ideas are closely-related if they are from the same conceptual domain, while two concepts are far-related if they are from discrepant or non-identical domains from human perspectives. The descriptions are in line with the ones in the study conducted by Han et al. (2018b). For instance, "sofa" and "chair", which belong to the same conceptual domain, are closelyrelated. "Sofa" and "soda" are far-related, as they belong to non-identical domains.

\subsection{Conceptual distance in combinational creativity}

A combinational idea is produced by combining a base idea (the basic idea of the combination) and an additive idea (the additional idea for creating the combination). Therefore, the conceptual distances between a base idea and an additive idea have significant effects on the degree of creativity of the combinational idea formed. Han et al. (2018b) have explored the conceptual distance in combinational creativity from design experts' perspectives using two studies. They suggested that far-related ideas (base and additive) could lead to outcomes that are more creative, comparing with closely-related ideas. They also indicated that far-related ideas are used more often in producing practical combinational designs.

In their first study, three design experts participated and evaluated two-hundred practical designs originated using combinational creativity. The combinational designs were decomposed into base and additive ideas according to the descriptions of the designs, respectively. In addition to base and additive ideas, names and images of the designs were also provided to give design experts more comprehensive information. Information from the two-hundred designs were constructed into a decision table, as shown in Figure 1. The table was then employed by the three design experts respectively for identifying the conceptual distances, whether far-related or closely-related, between the base ideas and the additive ideas. For the first study concerned, far-related ideas are employed more often, comparing with closely-related ones, for producing practical combinational designs. The designs originated using far-related ideas are called far-related combinational designs, and the ones produced using closely-related ideas are called closely-related combinational designs.

\begin{tabular}{|c|c|c|c|c|c|}
\hline No. & Name & Image & Base & Additive & $\begin{array}{l}\text { Closely-Related (Same Domain) (Type 'C') } \\
\text { Far-Related (Different Domains) (Type ' } F \text { ') } \\
\text { Others (Type ' } O \text { ', and please explain) }\end{array}$ \\
\hline 1 & Rule/One & & Pen & Ruler & c \\
\hline 2 & $\begin{array}{c}\mathrm{NI} \\
\text { Parasol }\end{array}$ & & Parasol & LED & $\mathrm{F}$ \\
\hline 3 & SWAN & & $\begin{array}{l}\text { LED } \\
\text { Light }\end{array}$ & Swan & $\mathrm{F}$ \\
\hline 4 & $\ldots$ & & $\ldots$ & -. & $\cdots$ \\
\hline
\end{tabular}

Figure 1. An example of a decision table used (Han et al., 2018b) 
The degree of creativity of combinational designs produced using far-related and closely-related ideas are explored in the second study. Ten designs in total, five far-related and five closely-related combinational designs, were selected randomly from the two-hundred practical designs for exploring which distance could lead to outcomes that are more creative. The designs selected had consensus agreements from the three design experts in the first study, being fully agreed as either far-related or closely-related combinational designs. Another two expert designers assessed the degree of creativity of the ten designs by using the CPSS (Creative Product Semantic Scale) questionnaire (Chulvi et al., 2012). Only the names, images, and descriptions of the ten designs were provided to the expert designers for assessment. For the second study concerned, the combinational designs produced by combining conceptually far-related ideas are more creative than the ones generated by employing closely-related ideas.

\section{Computational conceptual distance in combinational creativity}

The research conducted by Han et al. (2018b) has provided initial insights into how conceptual distances between ideas affects combinational creativity from a human perspective. It has indicated how to select appropriate base and additive ideas to produce combinational ideas based on conceptual distances. Theoretically, these findings could be implemented into the combinational based computational tools illustrated in the introduction section for improving the tools' effectiveness and efficiency. Using the Combinator (Han et al., 2018a) as an example, the tool can be programmed to have a higher rate of retrieving ideas which are far-related rather than closelyrelated for producing combinational prompts. However, the findings might not be applicable in computational tools, as software might be coded to have different assessment and 'understanding' of conceptual distances.

\subsection{Study methods}

In order to investigate conceptual distance in combinational creativity from a computational perspective, we have conducted a study utilising computational conceptual distance measurement tools. The distance measured by computational tools is regarded as computational conceptual distance in this paper. The same two-hundred combinational designs from the previous study conducted by Han et al. (2018b) are used in this paper to provide a comparison between the conceptual distances measured by expert designers and computational approaches. Also, the base and additive ideas used for originating the combinational designs had been identified in the previous study. The combinational designs were selected from the winners of international design competitions, such as Red Dot, by means by purposive sampling. The selected designs are all considered to be creative, as design competitions are generally creativity-oriented (Wang and Chan, 2010).

In this study, we have selected two computational conceptual distance measurement tools: ConceptNet (Speer et al., 2017) and WS4J (WordNet Similarity for Java). ConceptNet is a knowledge graph database connecting words and phrases with labelled and weighted edges (Speer et al., 2017). It contains knowledge from, for example, crowdsourcing and expert-created resources. ConceptNet is employed to help computers understand the meanings behind the words which people use. It can calculate the relatedness value of a particular pair of terms. This function is used in the study to measure conceptual distances computationally. WS4J is an online tool for several published semantic relatedness and similarity algorithms such as LESK (Leacock and Chodorow, 1998), WUP (Wu and Palmer, 1994), LCH (Leacock and Chodorow, 1998). WUP and LCH algorithms are used in the study to measure conceptual distances, respectively. WUP measures relatedness by calculating the depths of the two synsets in WordNet taxonomies as well as the depth of lexical conceptual structure (Wu and Palmer, 1994). LCH measures similarity based on the length of the shortest path between two synsets (Leacock and Chodorow, 1998).

\subsection{Results}

The conceptual distances between the base and additive ideas of the two-hundred combinational designs are measured using the WUP and LCH algorithms of the WS4J as well as ConceptNet, 
respectively. The measurement results of using ConceptNet, WUP and LCH are shown in Figure 2, 3 , and 4 , respectively. In the figures, the $\mathrm{x}$-axes represent the distance values and the $\mathrm{y}$-axes indicate the numbers of designs. For instance, there are 36 designs which are originated using base and additive ideas that are within the $(0.04,0.08]$ distance value range measured by ConceptNet, as shown in Figure 2. However, some of the distances between two ideas or words (phrases) could not be measured by the computational approaches, as the words are not contained in the databases employed by the approaches for conceptual distance measurements. These designs are regarded as invalid designs in this study. As a result, the ConceptNet measurement involves 196 valid designs, and both the WUP and LCH measurements include 193 valid designs.

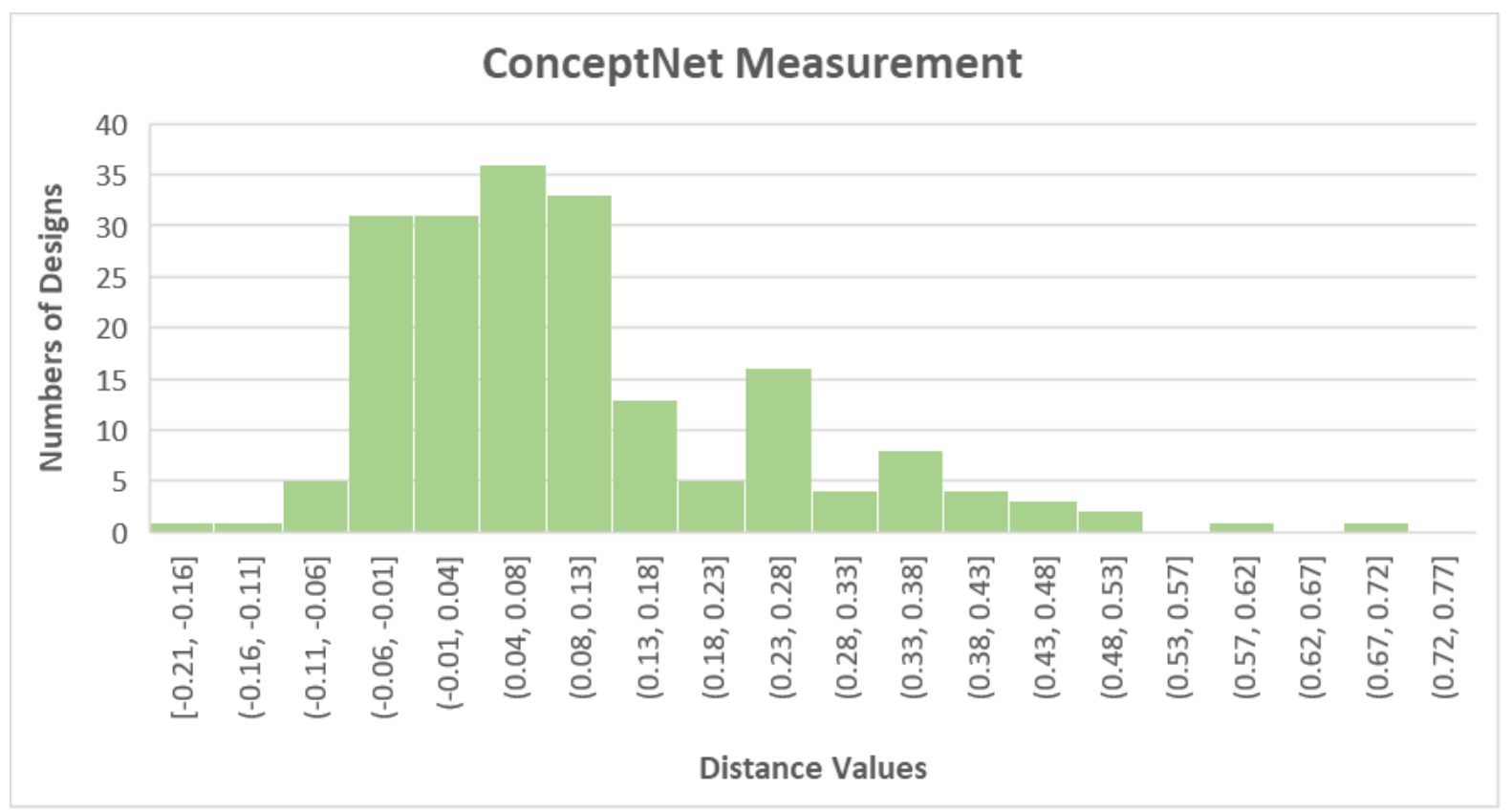

Figure 2. Conceptual distances measured by ConceptNet

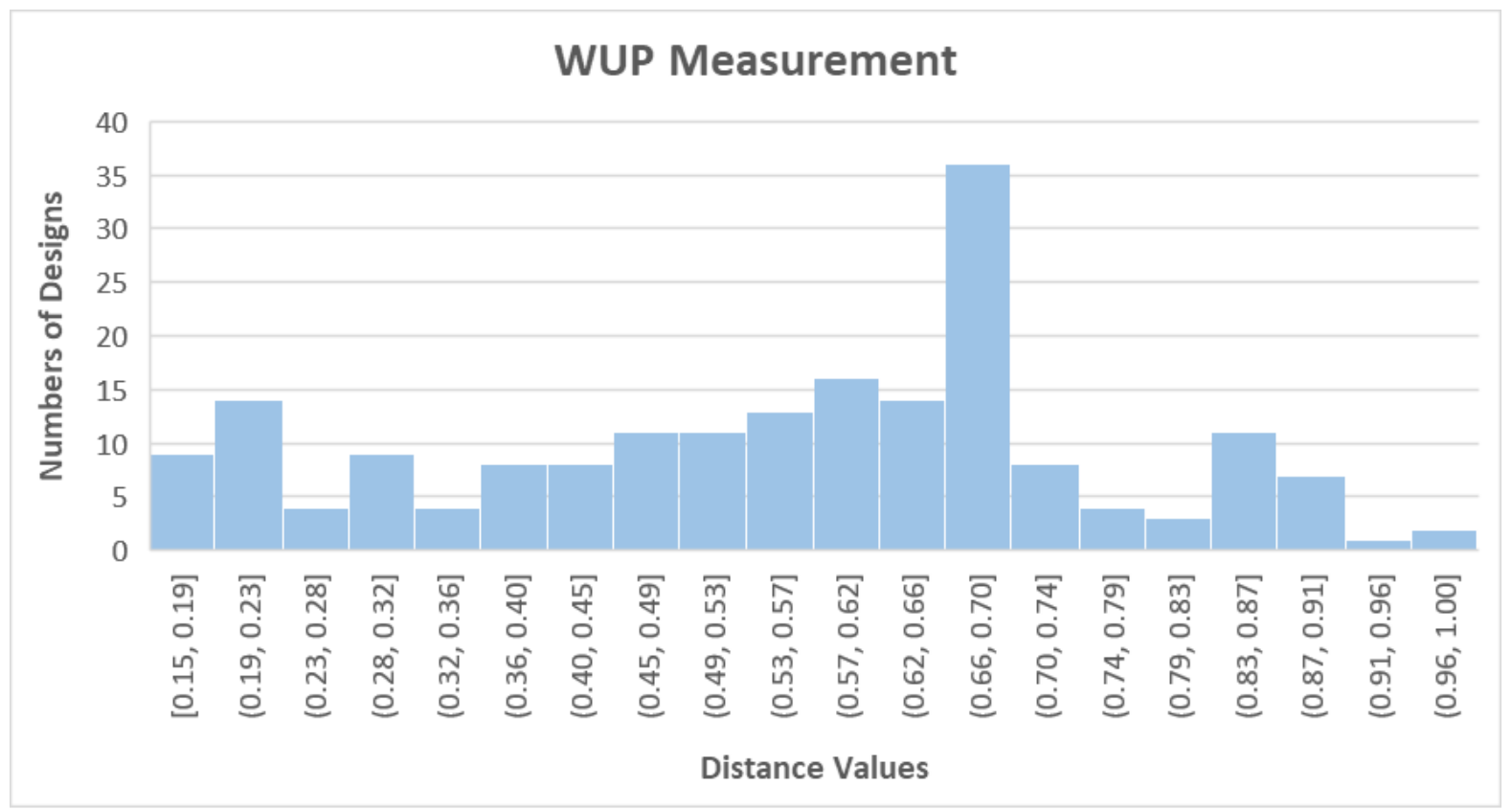

Figure 3. Conceptual distances measured by WUP 


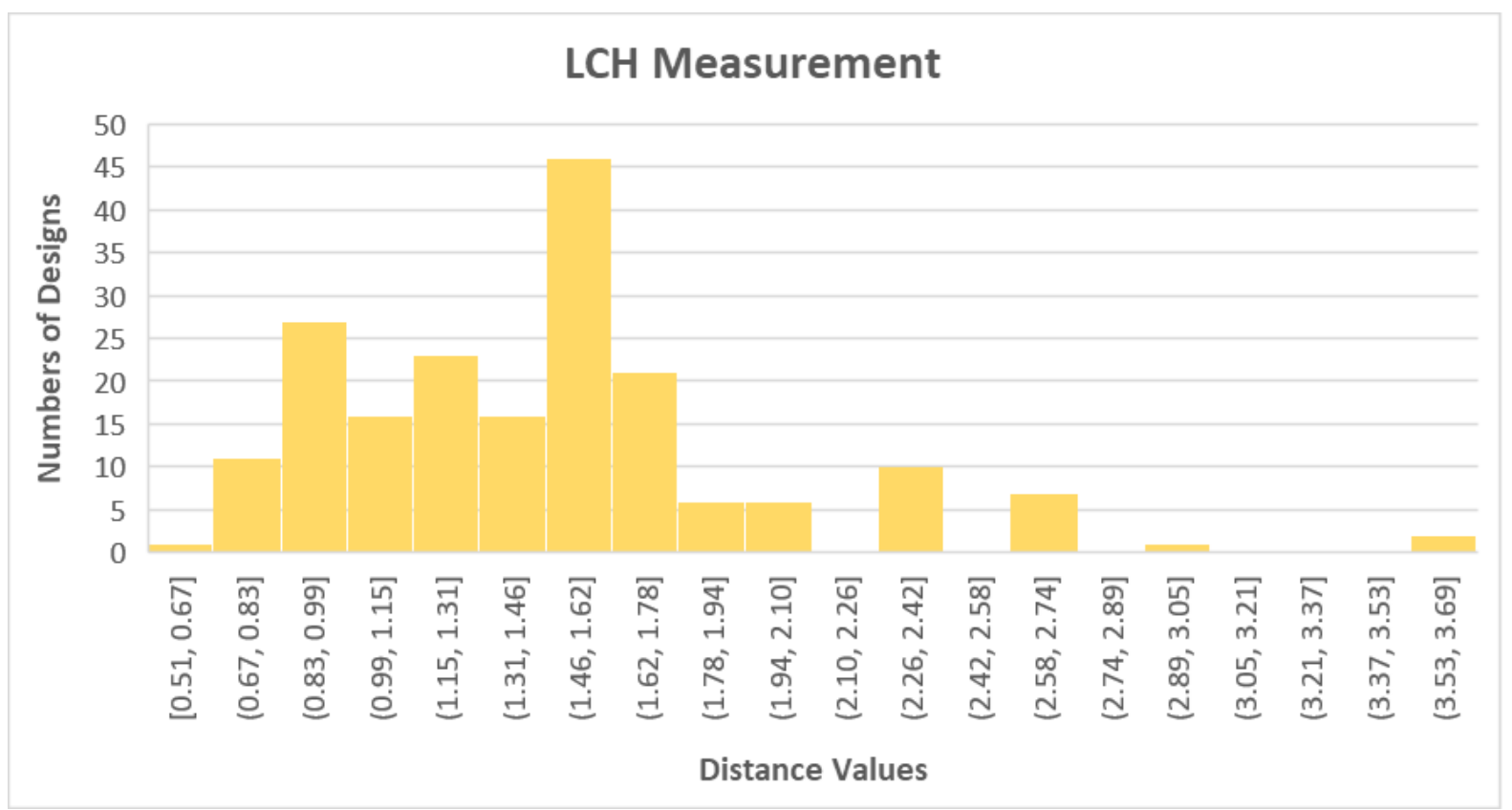

Figure 4. Conceptual distances measured by LCH

The distance values of ConceptNet measurement range from -1 to 1 , indicating very far to very close distances. The values of WUP measurement range from 0 (very far distance) to 1 (very close distance). The values of LCH measurement range from 0 to infinity, while a larger value shows a closer distance. Due to the different distance value ranges of the three measurements, all the three distance values axes are divided into 20 bins for providing a direct comprehension. As shown in the figures, the ConceptNet measurement and LCH measurement have indicated a similar trend that the majority of combinational designs belong to the lower distance value range. In comparison, the WUP measurement has demonstrated a more even distribution, with more designs belonging to the higher distance value range. However, it is challenging to identify whether a combinational is originated using far-related or closely-related ideas according to the distance values measured by the computational tools. Further analysis is conducted and illustrated in the next section

\section{Analysis and discussion}

A computational definition or description of conceptually far-related and closely-related distances is required. In this study, the mean value of the computational distance measurement is used as a demarcation point. We propose that distance values which are lower than the mean value indicate far-related distances, and the ones higher or equal to the mean value imply closely-related distances. In other words, a combinational design is considered a far-related design when the computationally measured conceptual distance between the base idea and the additive idea is lower than the mean distance, and vice versa.

The mean values, alternatively the demarcation points, of the distance values measured by ConceptNet, WUP and LCH are 0.11, 0.56 and 1.47, respectively. Based on the descriptions of far-related and closely-related distances above, ConceptNet, WUP and LCH have measured 123, 84 and 94 far-related designs, as well as 73, 109, and 99 closely-related designs, respectively. The computational measurements of the conceptual distances are compared with the expert designers' measurement, as shown in Table 1. The ConceptNet measurement has shown a similar result to the expert designers, of which a greater number of designs are originated using far-related ideas. However, more closely-related designs are identified using the WUP measurement, and a similar number of far-related and closely-related designs are identified using the $\mathrm{LCH}$ measurement. 
Table 1. Numbers of far-related and closely-related combinational designs - Expert designers, ConceptNet, WUP and LCH

\begin{tabular}{|c|c|c|c|c|}
\hline & Expert Designers & ConceptNet & WUP & LCH \\
\hline Far-Related Designs & 144 & 123 & 84 & 94 \\
\hline Closely-related Designs & 56 & 73 & 109 & 99 \\
\hline Invalid Designs & 0 & 4 & 7 & 7 \\
\hline
\end{tabular}

In addition to the analysis of the numbers of far-related and closely-related combinational designs above, we have conducted another assessment to analyse the degrees of creativity of far-related and closely-related combinational designs to provide further insights. The same ten combinational designs used in the previous study by Han et al. (2018b) are employed in this creativity assessment. As illustrated in section 2.2, five designs were considered far-related and the other five were considered closely-related by the three design experts in consensus agreements in the previous study. This assessment compares expert designers' measurement results and computational measurement results, as well as indicates which distance could lead to combinational designs that are more creative.

As shown in Table 2, the ConceptNet measurement has produced results in agreement with the expert designers. The LCH measurement results have shown similar results to the expert designers, of which LCH indicates Design 7 is a closely-related design while the expert designers had a different perception. In terms of the WUP measurement, the measurement results of Design 2 and Design 7 are different from the expert designers. Comparing the results of the three computational measurements, both WUP and LCH have indicated a different result against ConceptNet on Design 7. This could be caused by the different distance measurement algorithms used by the computational approaches, as well as the different databases employed. Besides, WUP has suggested a different measurement result on Design 2 in comparison with ConceptNet and LCH. However, all the conceptual distance values of the three computational measurements are close to their corresponding demarcation points, respectively. This implies that the conceptual distances of Design 2 calculated by the three computational measurements are neither far-related nor closely-related to some extent. Thereby, the descriptions of closely-related and far-related distances could be complemented with an additional moderately-related distance. Further studies are needed to suggest the demarcation points between far-related and moderately-related distances, as well as moderately-related and closely-related distances.

Table 2. Creativity scores and conceptual distances of the ten designs selected

\begin{tabular}{|c|c|c|c|c|c|}
\hline $\begin{array}{c}\text { Design } \\
\text { Number }\end{array}$ & $\begin{array}{c}\text { Creativity } \\
\text { Score }\end{array}$ & $\begin{array}{c}\text { Conceptual } \\
\text { Distance-Expert } \\
\text { Designers }\end{array}$ & $\begin{array}{c}\text { Conceptual Distance } \\
\text { - ConceptNet } \\
\text { (Value) }\end{array}$ & $\begin{array}{c}\text { Conceptual } \\
\text { Distance- WUP } \\
\text { (Value) }\end{array}$ & $\begin{array}{c}\text { Conceptual } \\
\text { Distance- LCH } \\
\text { (Value) }\end{array}$ \\
\hline $\mathbf{1}$ & 87 & Close & Close $(0.39)$ & Close $(0.75)$ & Close $(2.08)$ \\
\hline $\mathbf{2}$ & 109.5 & Far & Far $(0.09)$ & Close $(0.57)$ & Far $(1.39)$ \\
\hline $\mathbf{3}$ & 79.5 & Close & Close $(0.40)$ & Close $(0.87)$ & Close $(2.30)$ \\
\hline $\mathbf{4}$ & 68.5 & Close & Close $(0.36)$ & Close $(0.82)$ & Close $(2.08)$ \\
\hline $\mathbf{5}$ & 91 & Far & Far $(0.07)$ & Far $(0.22)$ & Far $(0.98)$ \\
\hline $\mathbf{6}$ & 77.5 & Close & Close $(0.48)$ & Close $(0.70)$ & Close $(1.74)$ \\
\hline $\mathbf{7}$ & 103.5 & Far & Far $(-0.02)$ & Close $(0.63)$ & Close $(1.61)$ \\
\hline $\mathbf{8}$ & 71.5 & Close & Close $(0.15)$ & Close $(0.64)$ & Close $(1.49)$ \\
\hline $\mathbf{9}$ & 80.5 & Far & Far $(-0.03)$ & Far $(0.48)$ & Far $(1.20)$ \\
\hline $\mathbf{1 0}$ & 101.5 & Far & Far $(-0.02)$ & Far $(0.18)$ & Far $(0.74)$ \\
\hline
\end{tabular}

The mean creativity scores of far-related and closely-related designs measured by different approaches are compared in Table 3. The mean creativity scores are calculated based on the creativity scores of the ten designs in Table 2 which were assessed by the two expert designers in 
the previous study. Cohen's $d$ is introduced as effect sizes to indicate the strength of the differences between the mean creativity scores of each measurement. As shown in the table, the mean creativity values of the far-related designs are higher than the closely-related ones with large effect sizes, in terms of expert designers' measurements. With regard to computational measurements, both the ConceptNet and LCH measurements have implied that far-related designs possess higher mean creativity scores than closely-related ones with large effect sizes. The LCH measurement has also indicated that far-related designs are more creative than closely-related designs, but with a moderate effect size. Thereby, the creativity assessment has indicated that far-related combinational designs are more creative than closely-related ones, for the selected combinational designs concerned.

Table 3. Mean creativity scores of far-related and closely-related combinational designs and effect sizes (Cohen's d) - Expert designers, ConceptNet, WUP and LCH

SD: Standard Deviation

(Cohen's d value and strength: 0.20: Small, 0.50: Moderate, 0.80: Large)

\begin{tabular}{|c|c|c|c|c|}
\hline & Expert Designers & ConceptNet & WUP & LCH \\
\hline Far-Related Designs (SD) & $97.2(10.26)$ & $97.2(10.26)$ & $91.00(8.57)$ & $95.63(10.92)$ \\
\hline Closely-related Designs (SD) & $76.8(6.46)$ & $76.8(6.46)$ & $85.29(14.57)$ & $81.25(11.57)$ \\
\hline Effect Sizes & 2.38 - Large & 2.38 - Large & 0.48 - Moderate & 1.28 - Large \\
\hline
\end{tabular}

\section{Conclusions}

To conclude, this paper has explored conceptual distances in combinational creativity from a computational perspective in the context of product design. The study conducted in the paper indicates that different computational measurements have yielded different results, of which the ConceptNet measurement has shown a more similar outcome to the expert designers comparing with WUP and LCH. This might be a result of the different algorithms and databases used by the computational measurements. In addition, it also suggests an introduction of an additional moderately-related distance to provide a better description of computational conceptual distances. However, further exploration is needed to produce more knowledge about this. For the study concerned, all the three computational measurements and the expert designers' measurement have indicated that far-related ideas could lead to combinational designs that are more creative comparing with closely-related ideas, albeit with a limited number of design samples. This paper has added to the understanding of combinational creativity in computational contexts. It could benefit design researchers in exploring and developing better computational design support tools which employ combinational creativity or the concept of 'combination'. A further study, which involves more design samples and more computational conceptual distance measurement tools, is planned to provide additional insights.

\section{References}

Blanchette, I. and Dunbar, K. (2000), "How analogies are generated: The roles of structural and superficial similarity", Memory \& Cognition, Vol. 28 No. 1, pp. 108-124. https://doi.org/10.3758/bf03211580

Boden, M.A. (2004), The creative mind: Myths and mechanisms, 2nd ed., Routledge, London, UK.

Boden, M.A. (2009), "Computer models of creativity”, AI Magazine, Vol. 30 No. 3, pp. 23-34. http://dx.doi.org/ 10.1609/aimag.v30i3.2254

Budanitsky, A. and Hirst, G. (2006), "Evaluating WordNet-based Measures of Lexical Semantic Relatedness", Computational Linguistics, Vol. 32 No. 1, pp. 13-47. https://doi.org/10.1162/coli.2006.32.1.13

Chan, J., Dow, S.P. and Schunn, C.D. (2015), "Do the best design ideas (really) come from conceptually distant sources of inspiration?", Design Studies, Vol. 36 No. Supplement C, pp. 31-58. https://doi.org/10.1016/ j.destud.2014.08.001

Chen, L. et al. (2019), “An artificial intelligence based data-driven approach for design ideation”, Journal of Visual Communication and Image Representation, Vol. 61, pp. 10-22. https://doi.org/10.1016/j.jvcir.2019.02.009

Chiu, I. and Shu, L.H. (2012), "Investigating effects of oppositely related semantic stimuli on design concept creativity", Journal of Engineering Design, Vol. 23 No. 4, pp. 271-296. https://doi.org/10.1080/0954 4828.2011.603298 
Chulvi, V. et al. (2012), "Assessment of the Relationships among Design Methods, Design Activities, and Creativity", Journal of Mechanical Design, Vol. 134 No. 11, pp. 111004-111011. https://doi.org/10.1115/ 1.4007362

Cooper, M.C. (2000), “Semantic Distance Measures”, Computational Intelligence, Vol. 16 No. 1, pp. 79-94. https://doi.org/10.1111/0824-7935.00106

Crilly, N. and Cardoso, C. (2017), "Where next for research on fixation, inspiration and creativity in design?", Design Studies, Vol. 50, pp. 1-38. https://doi.org/10.1016/j.destud.2017.02.001

Demirkan, H. and Afacan, Y. (2012), "Assessing creativity in design education: Analysis of creativity factors in the first-year design studio", Design Studies, Vol. 33 No. 3, pp. 262-278. https://doi.org/10.1016/j.destud. 2011.11.005

Fu, K. et al. (2013), "The Meaning of "Near" and "Far": The Impact of Structuring Design Databases and the Effect of Distance of Analogy on Design Output”, Journal of Mechanical Design, Vol. 135 No. 2, pp. 021007-021012. https://doi.org/10.1115/1.4023158

Gentner, D. and Smith, L.A. (2012), “Analogical reasoning”, In: Ramachandran, V.S. (Ed.), Encyclopedia of human behavior, 2nd ed., Elsevier, Oxford, pp. 130-136.

Georgiev, G.V., Sumitani, N. and Taura, T. (2017), "Methodology for creating new scenes through the use of thematic relations for innovative designs", International Journal of Design Creativity and Innovation, Vol. 5 No. 1-2, pp. 78-94. https://doi.org/10.1080/21650349.2015.1119658

Gray, J. and Rumpe, B. (2019), "Conceptual distance of models and languages", Software \& Systems Modeling, Vol. 18 No. 3, pp. 1571-1573. https://doi.org/10.1007/s10270-019-00734-z

Han, J., Forbes, H. and Schaefer, D. (2019a), "An Exploration of the Relations between Functionality, Aesthetics and Creativity in Design", Proceedings of the Design Society: International Conference on Engineering Design, Vol. 1 No. 1, pp. 259-268. https://doi.org/10.1017/dsi.2019.29

Han, J. et al. (2019b), "A Further Exploration of the Three Driven Approaches to Combinational Creativity", Proceedings of the Design Society: International Conference on Engineering Design, Vol. 1 No. 1, pp. 2735-2744. https://doi.org/10.1017/dsi.2019.280

Han, J. et al. (2019c), "Three driven approaches to combinational creativity: Problem-, similarity- and inspiration-driven", Proceedings of the Institution of Mechanical Engineers, Part C: Journal of Mechanical Engineering Science, Vol. 233 No. 2, pp. 373-384. https://doi.org/10.1177/0954406217750189

Han, J. et al. (2018a), "The Combinator - a computer-based tool for creative idea generation based on a simulation approach", Design Science, Vol. 4, p. e11. https://doi.org/10.1017/dsj.2018.7

Han, J. et al. (2018b), "The conceptual distances between ideas in combinational creativity", DS92: Proceedings of the DESIGN 2018 15th International Design Conference, pp. 1857-1866. https://doi.org/10.21278/ idc. 2018.0264

Leacock, C. and Chodorow, M. (1998), "Combining local context and WordNet similarity for word sense identification”, WordNet: An electronic lexical database, Vol. 49 No. 2, pp. 265-283.

Lofi, C. (2015), "Measuring Semantic Similarity and Relatedness with Distributional and Knowledge-based Approaches", Information and Media Technologies, Vol. 10 No. 3, pp. 493-501. https://doi.org/10.11185/ imt. 10.493

Lopez, R., Linsey, J.S. and Smith, S.M. (2011), "Characterizing the Effect of Domain Distance in Design-byAnalogy", In Proceedings of the ASME 2011 International Design Engineering Technical Conferences and Computers and Information in Engineering Conference, pp. 141-151. https://doi.org/10.1115/DETC2011-48428

Mohammad, S. (2008), "Measuring semantic distance using distributional profiles of concepts", Ph.D. Thesis, University of Toronto, Toronto, Canada.

Nagai, Y., Taura, T. and Mukai, F. (2009), "Concept blending and dissimilarity: Factors for creative concept generation process", Design Studies, Vol. 30 No. 6, pp. 648-675. https://doi.org/10.1016/j.destud.2009.05.004

Ozkan, O. and Dogan, F. (2013), "Cognitive strategies of analogical reasoning in design: Differences between expert and novice designers", Design Studies, Vol. 34 No. 2, pp. 161-192. https://doi.org/10.1016/j.destud. 2012.11.006

Resnik, P. (1999), "Semantic similarity in a taxonomy: an information-based measure and its application to problems of ambiguity in natural language", Journal of Artificial Intelligence Research, Vol. 11 No. 1, pp. 95-130.

Salahli, M. (2009), "An approach for measuring semantic relatedness between words via related terms", Mathematical and Computational Applications, Vol. 14 No. 1, pp. 55-63.

Sarkar, P. and Chakrabarti, A. (2011), “Assessing design creativity”, Design Studies, Vol. 32 No. 4, pp. 348-383. https://doi.org/10.1016/j.destud.2011.01.002

Simonton, D.K. (2017), "Domain-general creativity: On Generating Original, Useful, and Surprising Combinations", In: Kaufman, J.C., Glaveanu, V.P. and J, B. (Eds.), The Cambridge Handbook Of Creativity across Domains, The Cambridge University Press, Cambridge, UK, pp. 18-40. 
Speer, R., Chin, J. and Havasi, C. (2017), "ConceptNet 5.5: an open multilingual graph of general knowledge", Proceedings of the Thirty-First AAAI Conference on Artificial Intelligence, San Francisco, California, USA.

Taura, T. et al. (2007), "A Study on Design Creative Process Focused on Concept Combination Types in Comparison With Linguistic Interpretation Process", Proceedings of the 16th International Conference on Engineering Design ICED07.

Tversky, A. (1977), "Features of similarity”, Psychological Review, Vol. 84 No. 4, pp. 327-352. https://doi.org/ 10.1037/0033-295X.84.4.327

Wang, H.-H. and Chan, J.-H. (2010), An Approach to Measuring Metaphoricity of Creative Design, In: Taura, T. and Nagai, Y. (Eds.), Design creativity 2010, Springer London, London, pp. 89-96.

Ward, T.B. (1998), "Analogical distance and purpose in creative thought: mental leaps versus mental hops", In: Holyoak, K.J., Gentner, D. and Kokinov, B.N. (Eds.), Advances in analogy research: Integration of theory and data from the cognitive, computational, and neural sciences, New Bulgarian University Press, Sofia, Bulgaria, pp. 221-230.

Ward, T.B. (2011), “Analogies”, In: Runco, M.A. and Pritzker, S.R. (Eds.), Encyclopedia of creativity, 2nd ed., Academic Press, London, pp. 40-45.

Wu, Z. and Palmer, M. (1994), "Verbs semantics and lexical selection", Proceedings of the 32nd annual meeting on Association for Computational Linguistics, Las Cruces, New Mexico. https://doi.org/10.3115/ 981732.981751

Yang, H. and Zhang, L. (2016), "Promoting Creative Computing: origin, scope, research and applications", Digital Communications and Networks, Vol. 2 No. 2, pp. 84-91. https://doi.org/10.1016/j.dcan.2016.02.001 